\title{
Complementarity of LSST and WFIRST: Regarding Object Blending
}

W. A. Dawson, M. D. Schneider

February 13, 2014 
This document was prepared as an account of work sponsored by an agency of the United States government. Neither the United States government nor Lawrence Livermore National Security, LLC, nor any of their employees makes any warranty, expressed or implied, or assumes any legal liability or responsibility for the accuracy, completeness, or usefulness of any information, apparatus, product, or process disclosed, or represents that its use would not infringe privately owned rights. Reference herein to any specific commercial product, process, or service by trade name, trademark, manufacturer, or otherwise does not necessarily constitute or imply its endorsement, recommendation, or favoring by the United States government or Lawrence Livermore National Security, LLC. The views and opinions of authors expressed herein do not necessarily state or reflect those of the United States government or Lawrence Livermore National Security, LLC, and shall not be used for advertising or product endorsement purposes.

This work performed under the auspices of the U.S. Department of Energy by Lawrence Livermore National Laboratory under Contract DE-AC52-07NA27344. 


\section{Complementarity of LSST and WFIRST: Regarding Object Blending}

\section{W. A. Dawson \& M. D. Schneider (LLNL)}

Many aspects of the complementarity between LSST and WFIRST have been discussed in the WFIRST-AFTA Final Report (Spergel et al. 2013) and accepted within the community. For example, LSST's six optical filters $(u, g, r, i, z, y)$ and WFIRST's four infrared filters (Y,J,H,F184) ${ }^{1}$, which will achieve effectively the same depth, will be highly complementary for photometric redshift estimation. However, the important subject of object blending has been absent from many of these discussions. In this letter we wish to focus on the complementarity of LSST and WFIRST in specific regards to object blending issues. Both how blending will affect the naively assumed complementarity of the two surveys as well as how the complementarity of the two surveys can mitigate the detrimental effects of object blending.

\section{Blending: An Overview}

For this discussion we will limit ourselves to considering blending of objects in the LSST survey. LSST will be affected by atmospheric seeing (FWHM 0.7"), which will cause blended objects to be much more common than with the WFIRST survey (PSF 0.13"). In what follows it will suit us to define three types of blends:

1. Catastrophic blends are two or more blended objects that are overlap to such a degree that under normal reduction procedures (e.g. SExtractor) they would be considered a single object.

2. Conspicuous blends are two or more blended objects that overlap significantly, however normal reduction procedures are able to detect them as blended.

3. Innocuous blends are two or more objects that may overlap in the outer periphery of their isophotes, and thus are technically blended, but to such a small degree that the overlap has little to no effect on inferred properties of the two objects (e.g. shape, photometry, astrometry, etc.).

As we will see the primary driver for these three definitions is the fact that each population will affect the survey science goals differently and the mitigation strategy for each population will be different. Using a simple but educational formalism we can quantify the distinction between the three types of blends by just considering the separation of two galaxies ${ }^{2}$ represented by Gaussian profiles ${ }^{3}$. Then

\footnotetext{
${ }^{1}$ Filter complement proposed for the $200 \mathrm{deg}^{2}$ High Latitude Survey in Spergel et al. (2013). 2 We are only considering the case of blended galaxies. However the blending of stars with galaxies and other stars will become very important for lower galactic latitudes. Specific consideration of stellar blending will be saved for a later study.
} 
catastrophic blends will be represented by the case where the two galaxies are closer than the sum of the $1 \sigma$ distance from the center of each galaxy (see the top panel of Figure 1), innocuous blends will be represented by the case where two galaxies further apart than the sum of the 2s distance from the center of each galaxy (see the bottom panel of Figure 1), and conspicuous blends will occupy the region between. Figure 2 provides a more realistic example of the various types of blends as found in the comparison of HST and Subaru imaging.

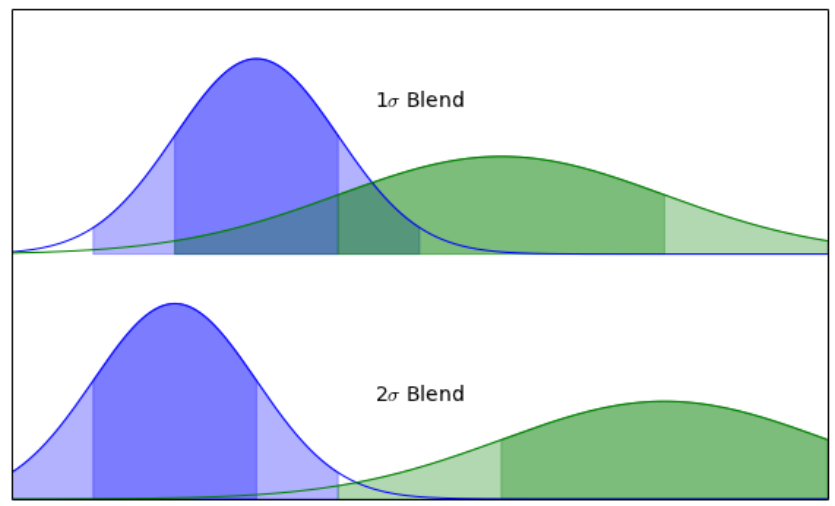

Figure 1: Gaussians representing the photometric profiles of two galaxies, with two examples illustrating the boundaries between Catastrophic and Conspicuous blends (upper panel), and Conspicuous and Innocuous blend (lower panel). Where objects closer than the $1 \sigma$ blend case in the upper panel are defined as catastrophic blends, objects further apart than the $2 \sigma$ blend case are defined as innocuous blends, and objects with separations between the bounds are defined as conspicuous blends.
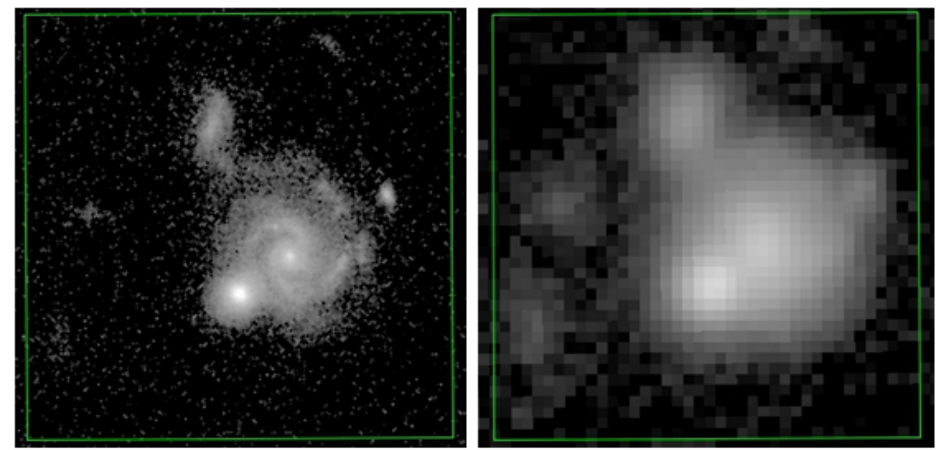

Figure 2: Examples of how objects as viewed from space (HST-ACS; left) can be blended to differing degrees in images from the ground (Subaru-Surpime Cam; right). In this image there are examples of catastrophic, conspicuous, and innocuous blends.

The fraction of blended objects will be a strong function of the limiting magnitude of the survey. To estimate the fraction of each type of blend as a function of limiting magnitude we convolved multiple HST-ACS survey images with a Gaussian PSF of 0.7 " FWHM (the average expected seeing of LSST). Knowing the "true" location of objects from the HST images enabled us determine when two objects became closer

3 This formalism neglects many important aspects of blending: relative magnitude, ellipticity, orientation, profile (e.g. sersic index), signal-to-noise, SED, pixilization effects, etc.. That said, the respective fraction of blends predicted by this simplistic formalism agrees well with detailed simulations. 
than a given $\sigma$ limit (as defined in Figure 1) after convolution with the 0.7" PSF. The approximate completeness limit of the HST surveys is 27 magnitudes (F775W, approximately LSST $i$-band), beyond which the fraction of blended objects will be underestimated. The GOODS fields (Giavalisco et al. 2004) are reasonable approximations of an average field in the LSST lensing survey area. The Musket Ball cluster field (Dawson et al. 2012) provides an example of an over-dense field, as it surveys a $\sim 5 \times 10^{14} \mathrm{M}_{\text {sun }}$ cluster system at $\mathrm{z}=0.53$.

Given that LSST will achieve a survey depth of 27.5 magnitudes (AB for a $5 \sigma$ point source detection), blending will affect a significant number of LSST sources. As can be seen from Figure 3, 15-20\% of the objects in the LSST lensing survey area will be catastrophic blends and $\sim 30-35 \%$ of the objects will be conspicuous blends. This means that blending will affect $\sim 45-55 \%$ of all LSST objects to a degree where it will pose a significant systematic to a number of science methods (e.g. weak lensing and SN measurements) and associated science goals (e.g. dark energy).

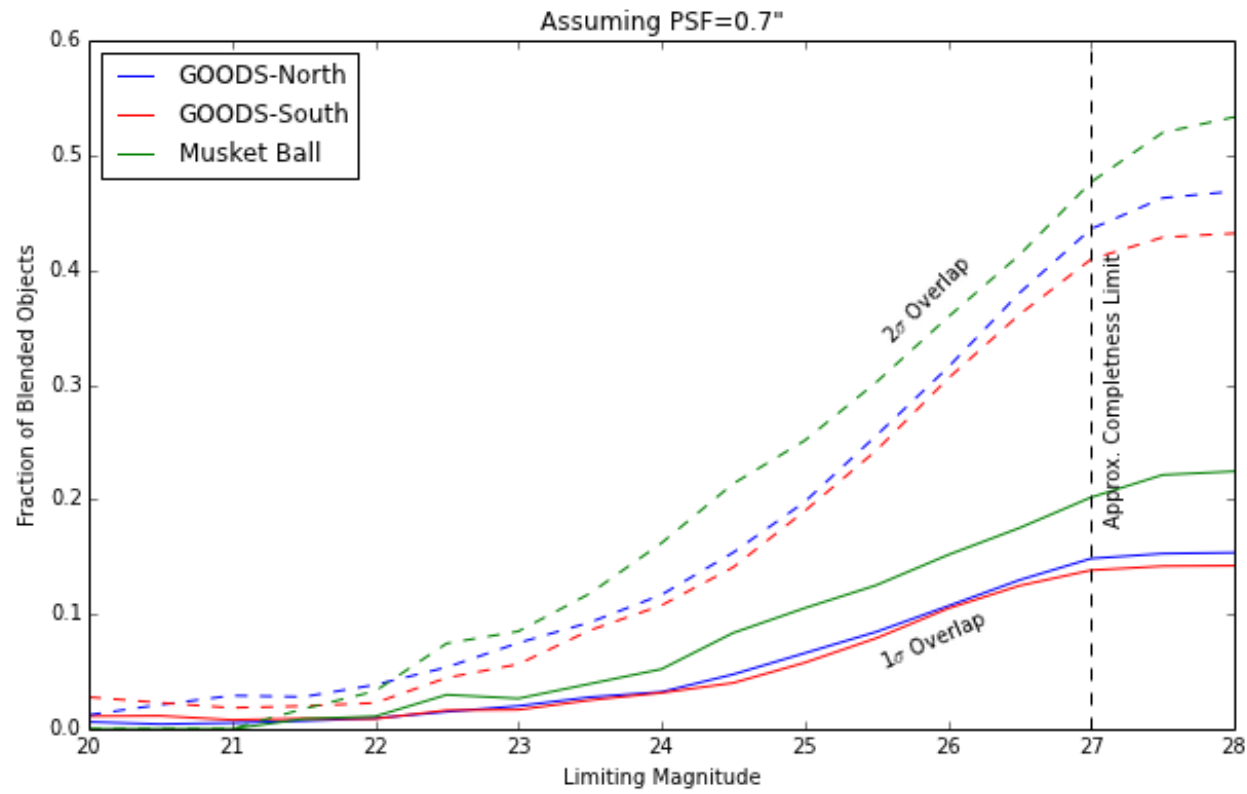

Figure 3: An estimation of the fraction of blended objects as a function of limiting magnitude (F775W, approximately LSST $i$-band) for all objects closer than the $1 \sigma$ overlap limit (solid lines) and all objects closer than the $2 \sigma$ overlap limit (dashed lines) assuming a ground based PSF of 0.7 ". These HST survey achieved an approximate completeness limit of 27 magnitudes, denoted by the vertical black dashed line, beyond which the estimated blend fraction will be under estimated.

\section{Blending: Impacts and Mitigation}

In this section we discuss the impact that catastrophic and conspicuous blends will have on various scientific measures critical to the dark energy science mission. We will also discuss how the complementarity of WFIRST can help mitigate the effects of these blends on LSST science. 
For the mitigation strategies we assume that a large fraction of the WFIRST survey area will be contained within the LSST survey footprint. However, we assume that WFIRST will overlap no more than $\sim 10 \%$ of the LSST survey. This is representative of the current WFIRST survey strategy. LSST blend mitigation would be significantly improved if there were more substantial overlap of the two surveys; however this might require significant compromises to the WFIRST independent science capability. During the upcoming formative years of each survey it is important for us to consider how to best optimize the complementarity of these surveys with respect to blending while maintaining their independent science capabilities.

\section{Blending characterization}

A statistical training approach based on the overlapping area of LSST and WFIRST is the overarching theme to the various blending mitigation methods suggested below. By identifying galaxy properties such as color, luminosity, and morphological characteristics we might identify clusters in this galaxy parameter space that statistically distinguish blends from non-blended objects. We could then apply this statistical classification to the entire LSST catalog. Figure 4 demonstrates a simple version of this classification approach.

Next step: Identify the galaxy properties that can be statistically informative about blending by studying our HST and Subaru imaging.

\section{Blending Impacts}

\section{Photometric Redshifts}

While photometric redshifts (photo-z's) do not provide a direct constraint on dark energy they are an integral component of almost all dark energy measures and will equally impact both LSST and WFIRST surveys. Thus they deserve special consideration.

It is also important to note that no existing photo-z method is designed to handle blended objects. Most photo-z algorithms depend on matching a galaxy's broadband photometry to expectations from template galaxies (either synthetic or a spectroscopic training sample). It is possible that blended objects will thus yield a larger fraction of 'catastrophic' outliers - errors in photo-z estimates of order one. This problem has not been addressed before because blending has not been important in shallower surveys, as demonstrated in Figure 3.

There are two fundamental concerns when considering the impact of blended objects on photo-z's. The first is the amount of flux contamination a particular object suffers from its blended partner ${ }^{5}$ and the second is the redshift difference of the blended objects.

\footnotetext{
${ }^{5}$ While we limit ourselves to a detailed discussion of photometric redshifts, it is important to note that that blending's more general impact on photometry, which will also impact
} 
Since photo-z's depend on the measured flux for a given object in each photometric band it is important to not only consider the spatial blending definitions of the previous section but also consider how blending affects the inferred flux of a given object. For example, two objects may be a catastrophic blend but if one object is much brighter than the other, then a reliable photo-z may be estimated for the brighter object. However, it is unlikely that a photo-z could be estimated for the faint object. For this reason it is important to assess the impact of a blend on an object by object basis. Kirkby (2013) introduced the definition of pixel purity $(P)$ for a given galaxy as

$$
P=\frac{\text { (signal from this galaxy) }}{(\text { total signal })}
$$

where he considers that each pixel in a detector will contain a total amount of signal (i.e. flux) from some number of objects. Thus for each pixel $i$ and object $j, P_{i j}$ can be defined. Using this basis we define flux contamination $(\Psi)$ of a given object to be:

$$
\Psi=1-\frac{\sum_{i=1}^{N_{\text {pixels }}} S_{i} P_{i}}{\sum_{i=1}^{N_{\text {pixels }}} S_{i}}
$$

where $S_{i}$ is the expected flux of the given object for pixel $i$ and $P_{i}$ is the pixel purity of the $i^{\text {th }}$ pixel. For negligible blending, e.g. when calculating $\Psi$ for a very bright object blended with a very faint object, or for the case of innocuous blends, $\Psi$ approaches zero. For significant blending, e.g. when calculating $\Psi$ for a faint object that is catastrophically or conspicuously blended with at bright object, $\Psi$ approaches 1 .

The impact of blends on photo-z's will also depend on the redshifts of the blended objects. If the two objects are at the same redshift (e.g. cluster or group members) then it should be expected that the blending will have minimal impact on the estimated photo-z's of the objects 6 . However, if the two objects are at very different redshifts and have significant flux contamination $(\Psi)$ then it is unreasonable to expect reliable photo-z estimates ${ }^{7}$.

large-scale structure interpretation, and some types of shear estimators, among other things.

${ }^{6}$ Even if one of the blended galaxies is star forming and the other is quiescent. At least to the degree that the photo-z method is capable of handling linear combinations of emission and absorption line spectral templates for a given redshift.

${ }^{7}$ At best one could hope that the photometric redshift probability density function might appear bimodal with peaks at the two galaxy redshifts 
The sensitivity of the photo-z estimate for a blended pair at different redshifts will be lessened to a degree by the typical photo-z uncertainty of galaxies in general, $\sigma_{j}=$ $\sigma_{\text {zphot }}\left(1+z_{j}\right)$, where $\sigma_{\text {zphot }} \sim 0.05$ for LSST. Since the photo-z resolution is limited to this degree it is reasonable to expect that when two blended objects have a redshift difference less than $\sim \sigma$ that the photo-z estimate of each of the blended objects will be minimally affected ${ }^{8}$. To quantify this we introduce the parameter $\zeta$, which is a measure of the redshift difference of two objects with respect to their photo-z uncertainty,

$$
\zeta=1-\frac{\int_{0}^{\infty} \frac{1}{\sigma_{1} \sqrt{2 \pi}} e^{\frac{-\left(z-z_{1}\right)^{2}}{2 \sigma_{1}^{2}}} \frac{1}{\sigma_{2} \sqrt{2 \pi}} e^{\frac{-\left(z-z_{2}\right)^{2}}{2 \sigma_{2}^{2}}} \mathrm{~d} z}{\left(\frac{1}{\sigma_{z_{\min }} \sqrt{2 \pi}}\right)^{2} \int_{0}^{\infty} e^{\frac{-\left(z-z_{\min }\right)^{2}}{\sigma_{z_{\min }}^{2}}} \mathrm{~d} z}
$$

This specific formalism assumes that the photo-z uncertainty of each of the blended galaxies ( 1 and 2 ) is represented by a Gaussian each at the location of the true redshift $\left(z_{1}\right.$ and $\left.z_{2}\right)$. Thus the numerator is the integral of their joint photo-z probability density functions (PDF). The denominator normalizes this function to the case where two galaxies are at the same redshift (specifically the redshift of the lower redshift object since, by definition of $\sigma_{j}=\sigma_{z p h o t}\left(1+z_{j}\right)$, this will have the tighter PDF and result in the maximum joint PDF integral for the two galaxies). Thus for galaxies at the same redshift (and same uncertainty $\left.{ }^{9}\right) \xi=0$, and for galaxies with large redshift separations, relative to their $\sigma, \zeta \rightarrow 1$.

Ultimately the impact of blending on the photo-z of a given galaxy will scale as some multiplicative combination of $\Psi$ and $\zeta$., see for example Figure 4 that plots $\sqrt{\Psi \cdot \zeta}$. For example, if a galaxy is blended with another galaxy but there is little flux contamination (either due to a large separation or that the contaminating galaxy is very faint) then $\Psi \sim 0$, and it won't matter if they are at very different redshifts, $\zeta \sim 1$. Similarly the photo-z will not be impacted significantly even if two objects are significantly blended, $\Psi \sim 1$, provided they are near the same redshift, $\zeta \sim 0$.

Next step: Estimate values for $\Psi$ and $\xi$ for the LSST galaxy population and the corresponding impact on the science. How well can we do when we do or do not know (statistically) about the blending-induced photo-z contamination?

\footnotetext{
8 This still should be proven.

${ }^{9}$ Note that this formalism can easily be extended to the case where the galaxies have more complex photo-z PDF's. However, for the purposes of this document it will suffice to limit our consideration to this simplistic case.
} 


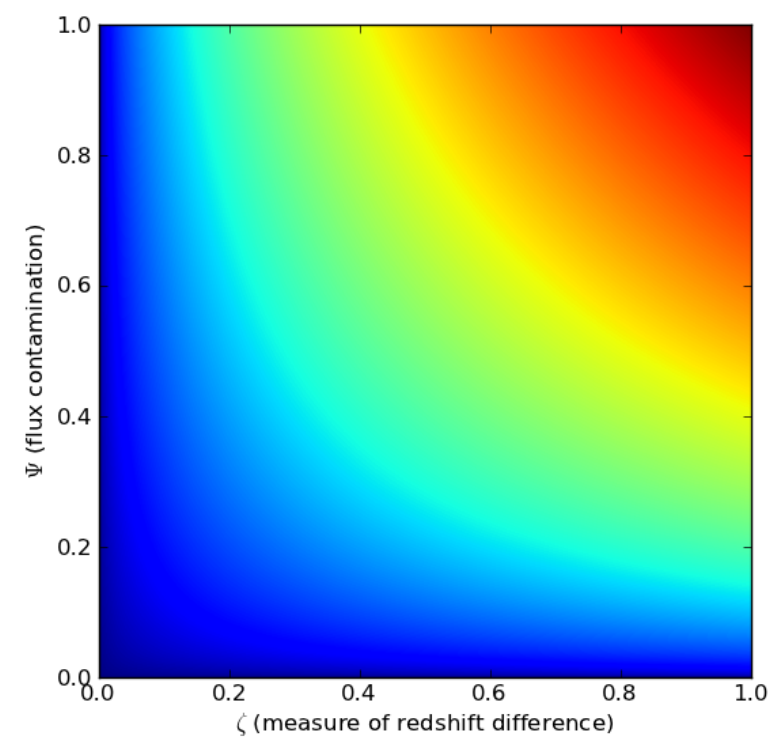

Figure 4: A potential blend photo-z impact measure, $\sqrt{\Psi \cdot \zeta}$. Blue represents minimal impact on the photo-z estimation with impact increasing towards red. Illustrating the concept that blending will have little impact on the photo-z estimate of a galaxy if it is blended with a galaxy at the same redshift $(\xi=0)$, regardless of the severity of the blend. Similarly for the case where two objects are at very different redshifts $(\xi \sim 1)$ but have minimal flux contamination $(\Psi \sim 0)$.

\section{Supernovae Science}

The SN science will also be sensitive to the amount of flux contamination $(\Psi)$ associated with blended objects. This is largely related to the fact that the SN science depends on having reliable estimates of the SN redshifts. Given that LSST will discover over ten million SN in the course of its ten-year survey (LSST Science Book), estimating redshifts of all these SN will be challenging. The only feasible means of estimating such a large number of redshifts is via broadband photometric measurements. These photometric redshifts will either be based on the assumed SN host galaxy, the SN itself, or some combination of the two. Blending will potentially be problematic for both.

SN photometric redshift estimates based on the host galaxy involve identifying a SN and associating it with pre/post-SN imaging of a galaxy (since the SN will influence the galaxy's photometry too much for a reliable photo-z estimate). Current estimates are that the average flux contamination of the LSST survey galaxies will be $\sim 6 \%$ in the R-band (Kirkby 2013) ${ }^{10}$. Assuming that the likelihood of a SN occurring at a location in a host galaxy is proportional to the flux surface density of that galaxy, then $\sim 6 \%$ of all SN will be blending confused (i.e. coming from the overlapping area of two galaxies). This is a conservative lower bound. For example, SN are more likely to be discovered in the outer regions of galaxies; thus the fraction of blending confused galaxies will be $\gtrsim 6 \%$. Also this ignores the scenario of catastrophic blends where the flux contamination on the outer halves of two blended galaxies

10 This is of order the number of SN-host mismatches observed in SDSS (5\%; Masao Sako private communication). While SDSS is not nearly as deep as LSST will be, thus the fraction should be lower, the SDSS PSF is larger. These effects should roughly balance each other. 
may be low; however the two galaxies are detected as one, thus confusing the true host. This scenario warrants further investigation since it is conceivable that LSST would otherwise benefit from simply flagging SN in conspicuous blends and excluding them from any analyses.

For these reasons, the method of estimating the redshift based directly on the SN broad-band photometry is attractive. This method is relatively young compared to photo-z's of galaxies, however there has been a number of promising studies (e.g. Asztalos et al. 2010, Kessler et al. 2009, Wang 2006, Wang et al. 2007, Kim and Miquel 2007). This method will avoid many of the complications associated with using the "host" galaxy photo-z. However, it will not entirely escape the complications of blending. Blending of background SN with foreground galaxies (the most likely scenario?) will increase the extinction of the SN. This extinction may vary significantly from assumed levels of extinction and can lead to biases in estimated $(1+\mathrm{z})$ at the few percent level (LSST Science Book).

Next step: Improve estimates of SN photo-z contamination due to blending and compare with the contamination when using host galaxy photo-z's either directly or as an additional prior on direct SN photo-z estimates.

\section{Weak Lensing Science}

Weak lensing (WL) measures will be responsible for providing some of the tightest constraints on the dark energy equation of state parameters. LSST's exact WL figure of merit will depend directly on our ability to correct an array of shape measurement systematic effects. One of the most dominant sources of WL systematic errors is multiplicative shape measurement bias due to convolution of the galaxy images with the atmospheric PSF. This bias acts to decrease the estimated ellipticities of galaxies, particularly for small sources of order the PSF (which make up the bulk of the WL source galaxies). Kasliwal et al. (2008) estimated this bias through the cross-correlation of the HST COSMOS survey with an overlapping Subaru-SuprimeCam survey. This cross-correlation is in many ways a representative precursor to the type of cross-correlation that will be possible between WFIRST and LSST. As expected Kasliwal et al. (2008) found that the bias increased with increasing magnitude and decreasing size. They then argued that drastic magnitude $(i<24.5)$ and size (half-light radii $>1.8$ ") cuts needed to be applied to reduce this bias to an acceptable level, leaving a galaxy surface density of 15 galaxies arcmin $^{-2}$ (insufficient for LSST's required science goals) ${ }^{11}$. Jee et al. (2013) measured a similar bias using simulations. However, they showed that the bias is stable and thus were able to correct for it rather than imposing strict sample cuts.

\footnotetext{
11 There are a number of improvements that should be made to this study before it is allowed to significantly influence future surveys. Such as the use of modern shape measurement methods applied to both space and ground data sets in a consistent manner, a more careful cross-matching algorithm, and accounting for blending. It is also unclear how they estimated the shear responsivity factor (e.g. Bernstein \& Jarvis 2002), which is designed to analytically correct known bias with ground based ellipticity measurements.
} 
Ideally LSST will implement a combination of both approaches. By cross-correlating LSST and WFIRST measured galaxy shapes we will be able determine the bias correction factor to apply to all LSST sources. Due to the large overlapping area and comparable depths we will be able to accurately determine this factor and highly tune it as a function of multiple galaxy properties (size, magnitude, color, type, etc.).

This method will be limited by both our ability to accurately cross-match galaxies between the two samples and the varying degrees of blending between the two surveys. Both will increase the rms scatter of the correction factor and potentially induce an additional bias. If two objects are incorrectly matched between the two catalogs then they will introduce a scatter of order the intrinsic ellipticity variance. The probability of incorrect cross-matching is a strong function of the degree of blending. We are currently developing a probabilistic cross-matching algorithm building upon Budavári \& Szalay (2008), which will provide a quantitative measure of the probability that two objects are uniquely matched between the catalogs (this will also prove valuable for the photo-z and SN complementarity of the two surveys). Blending will also increase the scatter of the estimated correction factor. Figure 5 illustrates how blending can cause catastrophic scatter in the ellipticity cross-correlation between space and ground based surveys.

Next step: Refine probabilistic cross-matching algorithm and repeat a Kasliwal et al. (2008) like analysis (with consistent/modern shape measurement algorithm) to quantify the stability of the correction factor estimation and its sensitivity to mismatches and blending.

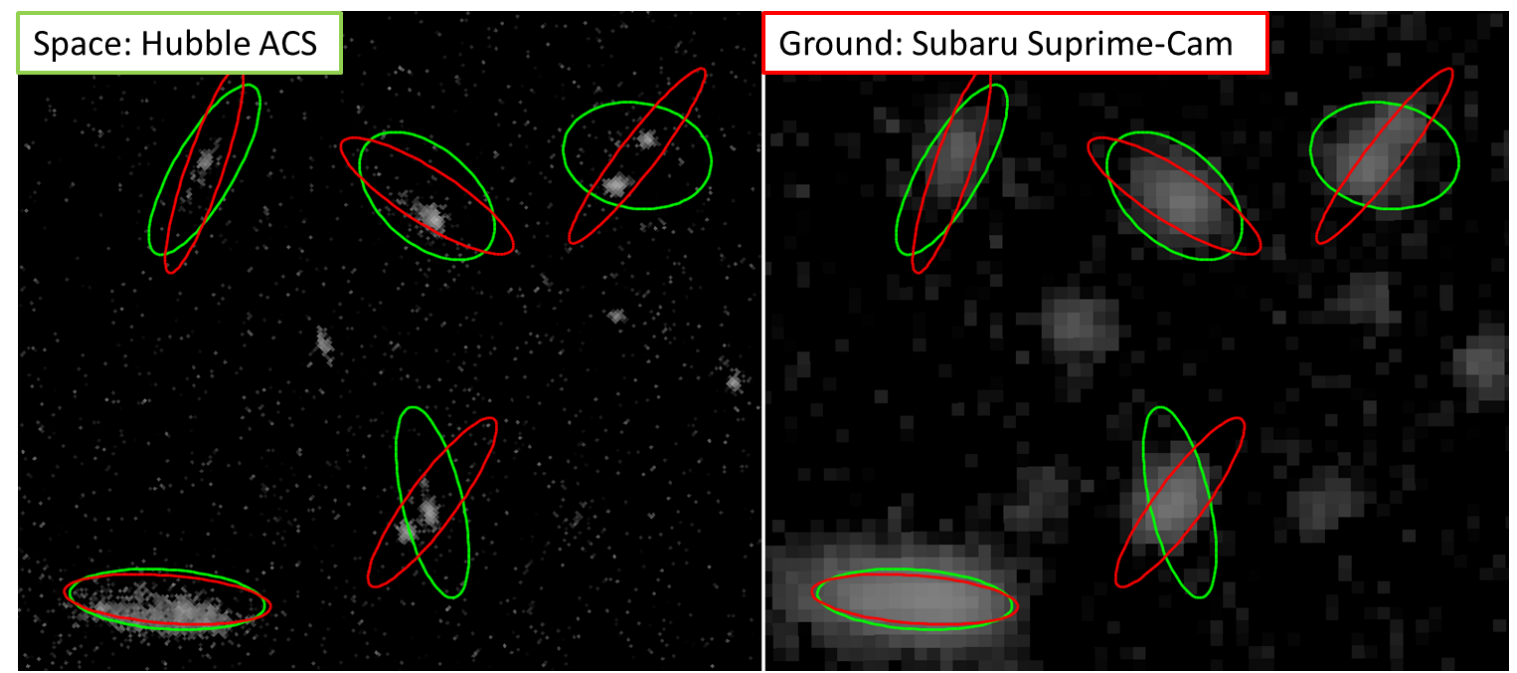

Figure 5: Comparison of HST-ACS (green) and Subaru Suprime-Cam (red) measured ellipticities for real observations of an 10 "x10". The image on the left is from two orbits with F814W and the image on the right is the same field but observed with $\sim 50$ minutes total of Subaru $i$-band with $\sim 0.6$ " seeing, the magnitude of the galaxies are $i \sim 25-26$. The galaxies were matched by selecting the HST galaxy closest to the Subaru galaxy center within a 1" radius. This comparison highlights how blending in the ground- 


\section{References}

Asztalos, S. et al., 2010. LOWER BOUNDS ON PHOTOMETRIC REDSHIFT ERRORS FROM TYPE Ia SUPERNOVA TEMPLATES. The Astrophysical Journal, 713(2), pp.1167-1173. Available at: http://labs.adsabs.harvard.edu/adsabs/abs/2010ApJ...713.1167A/.

Bernstein, G.M. \& Jarvis, M., 2002. Shapes and Shears, Stars and Smears: Optimal Measurements for Weak Lensing. The Astronomical Journal, 123(2), pp.583-618. Available at: http://adsabs.harvard.edu/abs/2002AJ...123..583B.

Budavári, T. \& Szalay, A.S., 2008. Probabilistic Cross - Identification of Astronomical Sources. The Astrophysical Journal, 679(1), pp.301-309. Available at: http://adsabs.harvard.edu/abs/2008ApJ..679..301B.

Dawson, W.A. et al., 2012. DISCOVERY OF A DISSOCIATIVE GALAXY CLUSTER MERGER WITH LARGE PHYSICAL SEPARATION. The Astrophysical Journal, 747(2), p.L42. Available at: http://adsabs.harvard.edu/abs/2011arXiv1110.4391D.

Giavalisco, M. et al., 2004. The Great Observatories Origins Deep Survey: Initial Results from Optical and NearInfrared Imaging. The Astrophysical Journal, 600(2), pp.L93-L98. Available at: http://adsabs.harvard.edu/abs/2004ApJ...600L..93G.

Jee, M.J. et al., 2013. COSMIC SHEAR RESULTS FROM THE DEEP LENS SURVEY. I. JOINT CONSTRAINTS ON $\Omega$ MAND $\sigma$ 8WITH A TWO-DIMENSIONAL ANALYSIS. The Astrophysical Journal, 765(1), p.74. Available at: http://adsabs.harvard.edu/abs/2013ApJ...765...74J.

Kasliwal, M.M. et al., 2008. A Comparison of Weak - Lensing Measurements from Ground - and Space - Based Facilities. The Astrophysical Journal, 684(1), pp.34-45. Available at: http://adsabs.harvard.edu/abs/2008ApJ...684...34K.

Kessler, R. et al., 2009. SNANA: A Public Software Package for Supernova Analysis. Publications of the Astronomical Society of the Pacific, 121(883), pp.1028-1035. Available at: http://adsabs.harvard.edu/abs/2009PASP..121.1028K.

Kim, A.G. \& Miquel, R., 2007. Measuring type Ia supernova distances and redshifts from their multi-band light curves. Astroparticle Physics, 28(4-5), pp.448-455. Available at: http://labs.adsabs.harvard.edu/adsabs/abs/2007APh....28..448K/.

Kirkby, David, 2013, Oct., 15th. Cosmic Shear Measurements in Deep Surveys. Presentation to LSST DESC Weak Lensing Telecon.

Spergel, D. et al., 2013. WFIRST-2.4: What Every Astronomer Should Know. eprint arXiv:1305.5425.

Spergel, D. et al., 2013. Wide-Field InfraRed Survey Telescope-Astrophysics Focused Telescope Assets WFIRSTAFTA Final Report. eprint arXiv:1305.5422.

Wang, Y., 2006. A Model-independent Photometric Redshift Estimator for Type Ia Supernovae. The Astrophysical Journal, 654(2), pp.L123-L125. Available at:

http://labs.adsabs.harvard.edu/adsabs/abs/2007ApJ...654L.123W/.

Wang, Y., Narayan, G. \& Wood-Vasey, M., 2007. Survey requirements for accurate and precise photometric redshifts for Type Ia supernovae. Monthly Notices of the Royal Astronomical Society, 382(1), pp.377-381. Available at: http://labs.adsabs.harvard.edu/adsabs/abs/2007MNRAS.382..377W/. 